\title{
Dynamics of energetic spectrum of solar-diurnal variations of cosmic rays in 19-24 solar activity cycles
}

\author{
Petr Yu. Gololobov ${ }^{\circledR}$, Vladislav G. Grigoryev, Germogen F. Krymsky, Sardaana K. Gerasimova \\ Correspondence \\ Yu.G. Shafer Institute of Cosmophysical Research and Aeronomy of SB RAS, Yakutsk, Russia, \\ gpeter@ikfia.ysn.ru
}

\section{OPEN ACCESS}

This work is published under the Creative Commons Attribution 4.0 International license (CC BY 4.0) Please note that individual, appropriately marked parts of the work may be excluded from the license may be excluded from the license other copyright conditions. other copyright conditions. If such third party material is not under the Creative Commons license, any copying, editing or public reproduction is only permitted with the prior consent of the respective copyright owner or on the basis of relevant legal authorization regulations.

\section{Keywords}

cosmic rays; solar diurnal variations; energy spectrum; neutron monitor; muon telescope

\begin{abstract}
The anisotropic angular distribution of cosmic rays (CR) in the interplanetary medium manifests itself on Earth as periodic diurnal intensity variations. Ground-based detectors of CRs have different energy sensitivity to the primary CR radiation and, therefore, the amplitude and phase recorded by them are also different. This fact makes it possible to study the energy spectrum of the variations when using a sufficient number of detectors. In this work, the results of the investigation of the energy spectrum of solar-diurnal variations of CRs obtained by a network of neutron monitors and muon telescopes are presented. The network allows measuring CRs with median energies from units to hundreds of $\mathrm{GeV}$. The expected values of the amplitude and phase of the daily $\mathrm{CR}$ variations at the selected ground-based stations for different types of the energy spectrum are shown. The calculated data are compared with experimental data for 19-24 solar activity cycles.
\end{abstract}

\section{Introduction}

The flux of galactic cosmic rays (CR) observed in near-Earth space is anisotropic. CR detectors located on Earth register such anisotropy as a periodic 24-hour variation of intensity, which is commonly called solar-diurnal variations (SDV). It is known that SDV reaches a maximum at 18:00 of local time and has an amplitude of about $0.5 \%$ of the total CR intensity. SDVs are observed in a wide range of energies and are found in the data of CR detectors located both high in the mountains and deep underground. At the same time, the SDV amplitude decreases with increasing energy and, according to neutron monitors (NM) data, is about 0.5\% (Munakata et al. 2014), and according to the underground muon telescope Matsushiro at a depth of $220 \mathrm{~m}$ w.e. (meters of water equivalent) - only $\sim 0.05 \%$ (Munakata et al. 2010). At the same time, the exact form of the SDV energy spectrum remains insufficiently studied so far. In Ahluwalia \& Ericksen (1971), Ahluwalia \& Riker (1987), Jacklyn et al. (1969) and Pomerantz \& Duggal (1971) an SDV rigidity spectrum was studied. For simplicity, we assume that CRs consist exclusively of protons. Then the rigidity spectra proposed in the above works can ber converted to the following form of the SDV energy spectrum

$$
f_{S D V}(E)=\left\{\begin{array}{c}
E^{\beta}, \text { if } E \leq E_{u} \\
0, \text { if } E>E_{u}
\end{array},\right.
$$


where the parameters $E_{u}$ and $\beta$ could be found by comparing the experimental data with theoretical calculations. The above studies showed that the spectrum most likely has the parameters $\beta$ $=0$ and $E_{u}=100 \mathrm{GeV}$, where the latter can vary depending on the solar activity cycle, reaching $50 \mathrm{GeV}$ at the minimum and $150 \mathrm{GeV}$ at the maximum. A later attempt (Hall et al. 1997) showed that $\beta$ also ranges from -0.3 to +0.5 . Also, Ahluwalia (1992) showed that $E_{u}$ has a functional relationship with the strength of the interplanetary magnetic field.

This work aims to study the SDV energy spectrum (1) using measurements of ground-based and underground CR detectors based on general ideas about the interaction of CRs with the geomagnetic field and the Earth's atmosphere. At the same time, the dynamics of the spectrum with the solar activity cycle is also considered.

\section{Determination of the SDV energy spectrum}

\subsection{Data and methods}

The list of ground-based CR detectors used in this work is presented in the table 1 . It includes 7 NMs located in high-, mid- and low-latitude regions of the Earth, as well as 7 ground-based and underground muon telescopes (MT). Such a set of detectors makes it possible to cover the region of median energies Emed from a few to hundreds of $\mathrm{GeV}$.

The CR intensity recorded by the i-th ground-based detector can be expressed as a series of spherical harmonics as follows:

$$
I^{i}=\sum_{n=0}^{\infty} \sum_{m=0}^{n}\left(a_{n}^{m} x_{n}^{m, i}+b_{n}^{m} y_{n}^{m, i}\right)
$$

where $x_{n}^{m, i}, y_{n}^{m, i}$ are components of the so-called receiving vector $\boldsymbol{R}_{n}^{m, i}, a_{n}^{m}$ and $b_{n}^{m}$ are the components of the multidimensional distribution vector of the CR $\boldsymbol{A}_{n}^{m}$ (Krymsky et al. 1966, 1967). In the framework of this work, we will limit ourselves to considering the equatorial components of the diurnal anisotropy, namely $a_{1}^{1}$ and $b_{1}^{1}$, since they are easiest to observe in an experiment.

\begin{tabular}{|c|c|c|c|c|c|c|}
\hline № & $\begin{array}{l}\text { Station name, } \\
\text { detector type }\end{array}$ & Abbreviation & $\begin{array}{l}\text { Geographical } \\
\text { coordinates }\end{array}$ & $\begin{array}{l}\text { Altitude, m / } \\
\text { depth, m w.e. } \\
\text { (water equivalent) }\end{array}$ & $\begin{array}{l}\text { Cutoff } \\
\text { rigidity, GV }\end{array}$ & $\begin{array}{c}\text { Emed, } \\
\text { GeV }\end{array}$ \\
\hline 1 & Apatity NM & APTY & $67.55 \mathrm{~N} 33.33 \mathrm{E}$ & 177 & 0.57 & 15 \\
\hline 2 & Climax NM & CLMX & $39.37 \mathrm{~N} \mathrm{106.18W}$ & 3400 & 2.99 & 11 \\
\hline 3 & Lomnicky stit NM & LMNC & $49.20 \mathrm{~N} 20.22 \mathrm{E}$ & 2634 & 3.98 & 13 \\
\hline 4 & Yakutsk NM & YKTK & $62.01 \mathrm{~N} 129.43 \mathrm{E}$ & 105 & 1.65 & 16 \\
\hline 5 & Thule NM & THUL & $76.50 \mathrm{~N} 68.7 \mathrm{~W}$ & 26 & 0.00 & 16 \\
\hline 6 & Rome NM & ROME & $41.90 \mathrm{~N} 12.52 \mathrm{E}$ & 60 & 6.32 & 24 \\
\hline 7 & Haleakala NM & HALE & $20.72 \mathrm{~N} 156.27 \mathrm{~W}$ & 3030 & 12.91 & 29 \\
\hline 8 & Nagoya MT & NAGO & $35.15 \mathrm{~N} 136.97 \mathrm{E}$ & 77 / 0 m w.e. & 11.50 & 52 \\
\hline 9 & \multirow{3}{*}{ Yakutsk MT } & Y00V & \multirow{3}{*}{$62.01 \mathrm{~N} 129.43 \mathrm{E}$} & $105 / 0$ m w.e. & \multirow{3}{*}{1.65} & 51 \\
\hline 10 & & Y07V & & $105 / 7$ m w.e. & & 69 \\
\hline 11 & & Y20V & & 105 / 20 m w.e. & & 104 \\
\hline
\end{tabular}




\begin{tabular}{|c|l|l|l|l|l|c|}
\hline 12 & Misato MT & MISA & $36.12 \mathrm{~N} 137.50 \mathrm{E}$ & $735 / 30$ m w.e. & 11.39 & 131 \\
\hline 13 & Sakashita MT & SAKA & $35.58 \mathrm{~N} 137.53 \mathrm{E}$ & $334 / 80 \mathrm{~m}$ w.e. & 11.50 & 270 \\
\hline 14 & Matsushiro MT & MATS & $35.15 \mathrm{~N} 136.97 \mathrm{E}$ & $408 / 220$ m w.e. & 11.50 & 672 \\
\hline
\end{tabular}

Table 1: Information about the NM stations and MT used in this work. Emed for MTs and NMs are determined based on response functions presented by Fujimoto et al. (1984) and Aleksan'yan et al. (1982).

As can be seen from equation (2), the amplitude and phase of the SDV observed by ground-based detectors will be determined by the vector $\boldsymbol{R}_{n}^{m, i}$. The calculation of the parameters $x_{n}^{m, i}$ and $y_{n}^{m, i}$ is carried out with some adaptation of the method of receiving vectors (Grigoryev et al. 2011, Yanchukovsky et al. 2016), in which the calculation results become similar to the coupling coefficients (Yasue et al. 1982; Fujimoto et al. 1984):

$$
x_{n}^{m}+i y_{n}^{m}=\frac{\int_{R c}^{\infty} \int_{0}^{\frac{\pi}{2}} \int_{0}^{2 \pi} W(E, \theta) f_{S D V}(E) N(\theta, \varphi) \sin \theta e^{i m \Psi} P_{n}^{m}(\sin \Phi) d E d \theta d \varphi}{\int_{R c}^{\infty} \int_{0}^{\frac{\pi}{2}} \int_{0}^{2 \pi} W(E, \theta) N(\theta, \varphi) \sin \theta d E d \theta d \varphi},
$$

where $W(E, \theta)$ are the response functions, $N(\theta, \varphi)$ is the directional diagram of the device, $\theta, \varphi$ are latitude and longitude, $\Psi$ and $\Phi$ are the asymptotic angles of arrival of particles. Here, the integral in the denominator is equal to the total CR intensity, and in the numerator - the intensity due to the SDV. Therefore, the amplitudes and phases of diurnal variations observed by ground-based detectors will be determined by the expressions

$$
A_{1}^{i}=\sqrt{\left(a_{1}^{1}\right)^{2}+\left(b_{1}^{1}\right)^{2}} \cdot \sqrt{\left(x_{1}^{1, i}\right)^{2}+\left(y_{1}^{1, i}\right)^{2}} \text { and } P_{1}^{i}=\operatorname{atan}\left(a_{1}^{1} / b_{1}^{1}\right)-\operatorname{atan}\left(x_{1}^{1, i} / y_{1}^{1, i}\right) \text {, respectively. }
$$

\subsection{Analysis of the obtained results and discussion}

Assuming an 18-hour maximum anisotropy and specifying the distribution components as $a_{1}^{1}=1$ and $b_{1}^{1}=0$, we investigate the dependence of $A_{1}^{i}$ and $P_{1}^{i}$ on the spectrum parameters $\beta$ and $E_{u}$ in (1). Considering $-1.0 \leq \beta \leq 1.0$ we get the picture shown in figure $1 \mathrm{a}$, $\mathrm{b}$. As you can see, with negative values of $\beta$, the amplitudes of daily variations observed by NMs significantly exceed MT, but with an increase of $\beta$ the situation changes in the opposite direction. Only when $\beta$ is close to 0 the expected amplitudes for NM and MT are mutually comparable. The difference between phases $P_{1}^{i}$ of MTs and NMs is practically leveled when $\beta>0$, and, on the contrary, it grows when $\beta<0$. Comparing this picture with the SDV parameters measured experimentally in 1990-1998 (figure 1 e, f), we can conclude that $\beta$ should vary near 0 .

Based on the foregoing, consider the dependence of the same parameters $A_{1}^{i}$ and $P_{1}^{i}$ on $E_{u}$ at $\beta=0$ (figure 1c, d). It is seen that, as a result of the influence of the geomagnetic field and the Earth $s$ atmosphere, the primary SDVs are suppressed and phase is shifted at an early time. Moreover, $A_{1}^{i}$ observed for MT is always lower than for NM. On the other hand, the $P_{1}^{i}$ phase is higher for mid- and low-latitude stations than for high-latitude stations, regardless of the type of detector. It is noteworthy that the $A_{1}^{i}$ and $P_{1}^{i}$ of NMs strongly depend to $E_{u}$ only within $\sim 100 \mathrm{GeV}$, while $A_{1}^{i}$ and $P_{1}^{i}$ of MTs - within $\sim 400 \mathrm{GeV}$. 

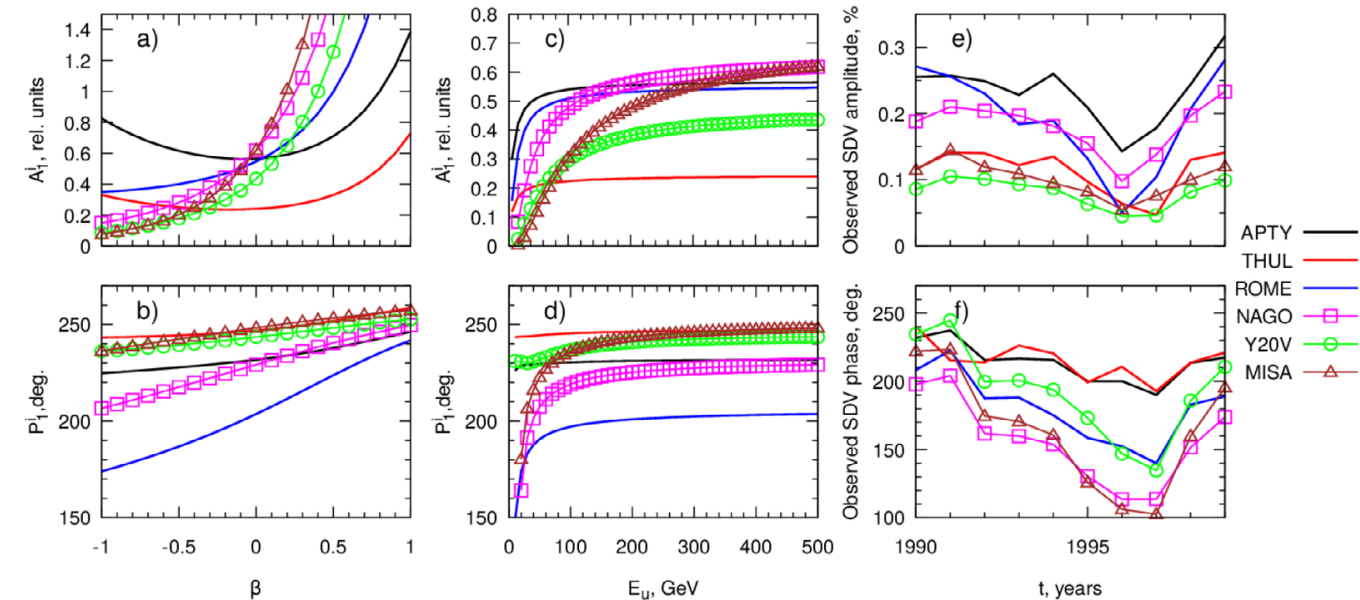

Figure 1: $(\mathrm{a}, \mathrm{b})-A_{1}^{i}$ and $P_{1}^{i}$ expected for different values of in equation (1); (c, d) - $A_{1}^{i}$ and $P_{1}^{i}$ expected for different values of $E_{u}(\beta=0) ;(\mathrm{e}, \mathrm{f})$ - amplitude and phase of SDV measured by ground-based detectors (see table 1). To facilitate the perception of the picture, only a part of the detectors are presented.

The shown above result qualitatively demonstrates the applicability of the theoretical spectrum in describing experimental data. For a quantitative assessment of the effect, we consider the ratios: $A_{1}^{i} / A_{1}^{0}$ and $P^{i}=P_{1}^{i}-P_{1}^{0}$, where we will take APTY as the 0th device, due to its stability and continuity. These relationships, expected theoretically and obtained experimentally, are presented in figure 2. The figure demonstrates the relationship between $E_{u}$ and the level of solar activity. At the same time, the $E_{u}$ values are quite consistent with the previously obtained results of other 50 authors $\left(\mathrm{GeV} \leq E_{u} \leq 150 \mathrm{~V}\right)$. However, $E_{u}$ cannot be determined unambiguously.
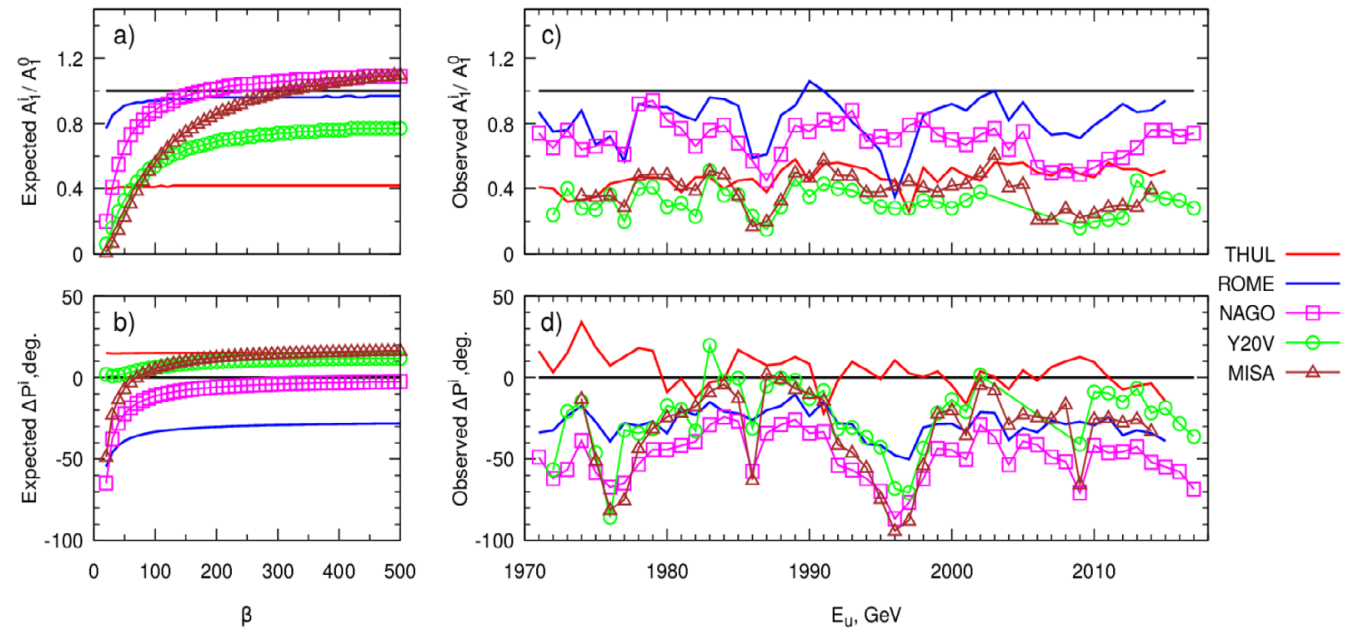

Figure 2: $(\mathrm{a}, \mathrm{b})$ - calculated values of $A_{1}^{i} / A_{1}^{0}$ and $\Delta P^{i}$ expected for different values of $E_{u}(\beta=0)$. (c, d) - the same parameters obtained experimentally for the period 1971-2017. 
Figure 3 shows the values of the amplitude of the SDV according to the measurements of CR detectors presented in table 1, as well as, for comparison, the same parameters expected from theory. The experimental SDV was obtained separately for periods of maximum and minimum solar activity through averaging of the SDV amplitudes observed in 1980, 1990, 2000, 2013 and 1976, 1986, 1996, 2008, respectively. It can be seen that there is a mutual agreement between the experiment and theory. A closer look shows that there are some disagreements. The probable reason is the greatly simplified form of the SDV spectrum, as well as the lack of accounting of the temperature effect (for MT) and the orbital motion of the Earth (Compton-Getting effect). Also, from the figure, it is seen that the SDV amplitudes observed by LMNC and CLMX during solar activity maximum greatly differ from the estimated values. These stations are high-altitude stations and, probably, the response functions need to be estimated more precisely.

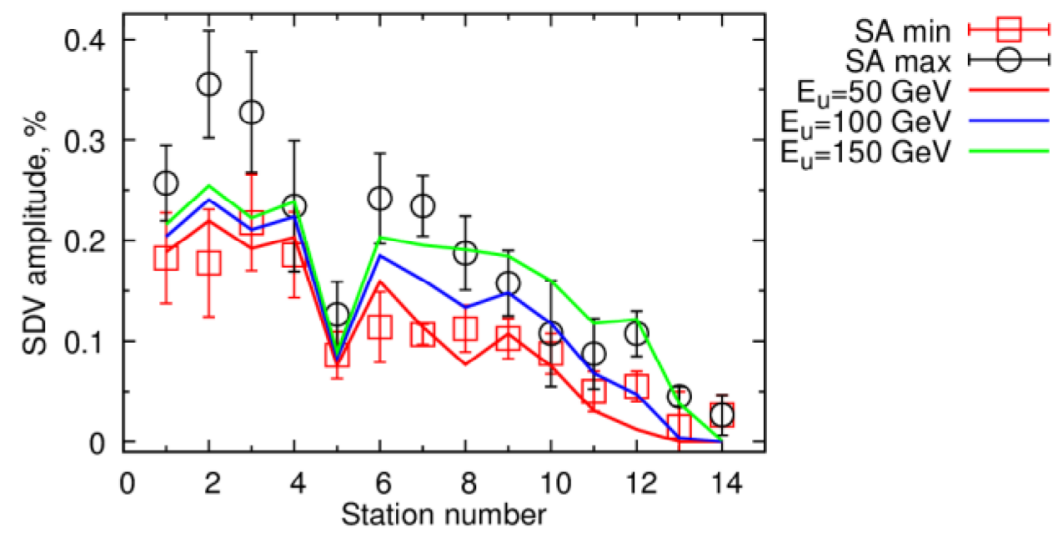

Figure 3: Expected values (solid colored curves) and experimentally observed SDV amplitudes (points) during periods of minima and maxima of solar activity. The calculation was performed at $E_{u}(\beta=0)=50,100$, $150 \mathrm{GeV}$. Station numbers correspond to those presented in the table 1 . The errors deduced from the dispersion of the mean monthly values.

\section{Conclusion}

Based on NM data and ground and underground MT data, the energy spectrum of SDV was studied. The measured SDV values were compared with those theoretically expected on the selected CR detectors for the period 1971-2017. It is shown that the spectrum of a flat form with an upper cutoff $\sim 100 \mathrm{GeV}$ is qualitatively describing the experimental data. More research is needed to determine the exact shape of the energy spectrum.

\section{Acknowledgments}

The work is carried out with partial support of the ShICRA Basic Research Program no. AAAAA17-117021450058-6 and the RFBR grants Nos. 18-42-140002-r_a, 18-02-00451-a.

We thank WDC for Cosmic Rays ${ }^{1}$, GMDN team ${ }^{2}$ and Cosmic-Ray section of Solar-Terrestrial environment laboratory of Nagoya University ${ }^{3}$ for providing their data.

1 http://cidas.isee.nagoya-u.ac.jp/WDCCR/index.html (last accessed April 20, 2021).

2 http://cosray.shinshu-u.ac.jp/crest/DB/Public/Archives/GMDN.php (last accessed April 20, 2021).

3 http://cosray.shinshu-u.ac.jp/crest/DB/Public/main.php (last accessed May 31, 2021). 


\section{References}

Ahluwalia, H.S., 1992, Geophys. Res. Lett. 19, 633, DOI: https://dx.doi.org/10.1029/92GL00525

Ahluwalia, H.S., Ericksen, J.H., 1971, JGR. 76, 6613, DOI: https://dx.doi.org/10.1029/JA076i028p06613

Ahluwalia, H.S., Riker, J.F., 1997, Planet. Space Sci. 35, 39, DOI: https://dx.doi.org/10.1016/0032-0633(87)90142-5

Aleksan'yan, T.M., Dorman, I.V., Dorman, L.I. et al., 1982, Izv. Akad. Nauk SSSR Ser. Fiz., 46, 1689 (in Russian)

Fujimoto, K., Inoue, A., Murakami, K., 1984, Rep. Cosmic Ray Res. Lab., No.9, 185

Grigoryev, V.G., Starodubtsev, S.A., Krymsky, G.F., et. al., 2011, Proc. 32nd ICRC. 11, 252, DOI: https://dx.doi.org/10.7529/ICRC2011/

V11/0360

Hall, D.L., Duldig, M.L., Humble, J.E., 1997, ApJ. 42, 1038, DOI: https://dx.doi.org/10.1086/304158

Jacklyn, R.M., Duggal, S.P., Pomerantz, M.A., 1969, Proc. 11th ICRC, 29, 47, ed. A. Somogyi, Acta Physica

Krymsky, G.F., Kuz'min, A.I., Chirkov, et al., 1966, Geomag. Aeron. 6, 991 (in Russian)

Krymsky, G.F., Kuz'min, A.I., Chirkov, et al., 1967, Geomag. Aeron. 7, 7 (in Russian)

Munakata, K., Mizoguchi, Y., Kato, C., et al., 2010, ApJ. 712, 1100, DOI: https://dx.doi.org/10.1088/0004-637X/712/2/1100

Munakata, K., Kozai, M., Kato, C., et al., 2014, ApJ. 791, 22, DOI: https://dx.doi.org/10.1088/0004-637X/791/1/22

Pomerantz, M.A., Duggal, S.P., 1971, Space Sci. Rev. 12, 75, DOI: https://dx.doi.org/10.1007/BF00172130

Yanchukovsky, V.L., Grigoryev, V.G., Krymsky, G.F. et. al., 2016, Solar-Terrestrial Phys. 2, 103, DOI: https://dx.doi.org/10.12737/16762 